\title{
IRA Code Design for Iterative Detection and Decoding: A Setpoint-based Approach
}

\author{
F. Lenkeit, C. Bockelmann, D. Wübben, A. Dekorsy \\ Department of Communications Engineering \\ University of Bremen, Germany \\ Email: \{lenkeit, bockelmann, wuebben, dekorsy\}@ant.uni-bremen.de
}

\begin{abstract}
In this paper, a novel setpoint-based design approach for Irregular Repeat Accumulate (IRA) codes in iterative detection and decoding structures is presented. In contrast to conventional IRA code design in which the convolutional decoder is combined with the detector, the goal behind this approach is to keep the IRA decoding structure consisting of convolutional decoder and repetition decoder intact, i.e. to consider it as an inner loop of the overall detection structure. The outer loop is then composed of the IRA decoder and the system specific detector. This approach requires to adapt the irregular repetition code jointly to the convolutional decoder as well as to the detector which is achieved by formulating setpoints for the inner and outer code characteristic. As will be shown, the presented code design approach, although starting from a completely different viewpoint as the conventional approach, leads to an irregular repetition code with a very similar transfer characteristic and code rate than the conventional approach.
\end{abstract}

\section{INTRODUCTION}

In 1993, Berrou, Glavieux and Thitimajshima published their work on a class of what they called turbo-codes [1]. Due to the concatenation of simple component codes connected via interleavers they achieved error-free transmission near the capacity limit while still allowing decoding at resonable effort. Divsalar et. al. presented a generalization of turbo-codes with what they called repeat-accumulate (RA) codes [2] consisting of a serial concatenation of an outer repetition code and an inner accumulator. Like turbo-codes, RA codes offer a linear encoding complexity and allow an efficient decoding based on belief propagation. In [3] Jin et. al. generalized RA codes to so-called Irregular Repeat-Accumulate (IRA) codes applying a mixture of repetition codes of different code rates as outer component code. Due to this code mixture, IRA codes offer higher degrees of freedom than regular RA codes, making them an interesting candidate for verious applications.

A particular interesting field of application for IRA codes are iterative detection schemes found in Multiple-Input Multiple-Output (MIMO)- or in Multi-User-Detection (MUD) systems. In such systems, code design requires thorough adaption of the code parameters to the overall detector, e.g. a sphere decoder in MIMO systems or a soft-RAKE detector in Interleave-Division Multiple-Access (IDMA) systems [10].

This work has been performed in the framework of the FP7 project ICT317669 METIS, which is partly funded by the European Union. The authors would like to acknowledge the contributions of their colleagues in METIS, although the views expressed are those of the authors and do not necessarily represent the project.
Both detectors realize a sub-optimal implementation of the maximum a-posteriori-probability (MAP) detector. For IRA codes this means, that the irregular repetition code needs to be adapted to the convolutional decoder as well as to the detector at the desired working point.

In [4], ten Brink and Kramer presented a general design approach for IRA codes in the context of iterative detection systems. There, they combined the detector with the convolutional decoder to an inner entity whose transfer characteristic was determined by EXIT-analysis [5]. The degree distribution of the irregular repetition code was then found by matching the EXIT-curve of the irregular repetition code to the EXIT-curve of the inner entity. This methodology was adapted successfully for IDMA-based multi-user systems, e.g., in [6] and later in [7].

Despite the success of the aforementioned approach it seems more intuitive to keep the decoding structure of the IRA code consisting of convolutional decoder and repetition decoder intact, i.e. to treat the IRA decoder as inner entity. However, doing so requires to adapt the repetition code jointly to the convolutional decoder as well as to the detector. The goal is then to end up with an IRA code that fulfills the following two properties:

1) in the beginning of the iterative detection process, the code should only slightly improve the overall detection with every outer iteration. It should not instantly lead to a perfect decoding as this would implicitly mean a rate loss.

2) towards the end of the iterative detection process, i.e. with sufficient information from the detector, the code should allow perfect decoding.

In the following, we present a design approach for IRA codes in the context of iterative detection schemes which treats the IRA decoder as inner entity and allows to design the code such that the two aforementioned properties are fulfilled. Hereby, we focus on non-systematic IRA codes as in [4] which can perform as well as systematic IRA codes while simplifying the design process, since the repetition decoder only receives information from the convolutional decoder and not from the detector. In order to optimize the repetition code jointly to the detector and the convolutional decoder, a desired outer code characteristic is formulated which is matched to the detector. From this desired characteristic a set of setpoints is determined which the code has to achieve. Translating these setpoints to 


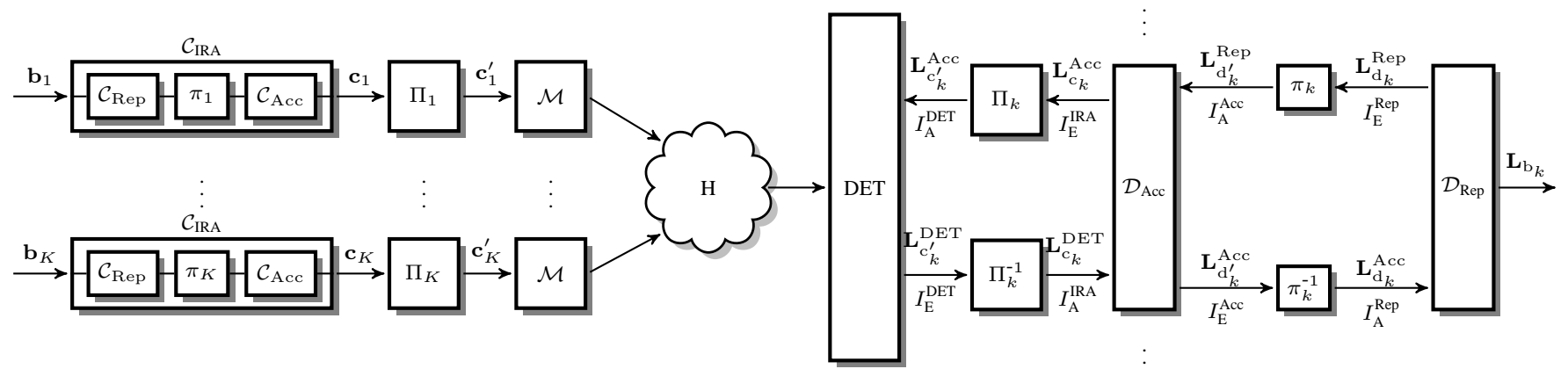

Fig. 1. Communications chain with source(s) consisting of $K$ parallel identical IRA channel encoders $\mathcal{C}_{\text {IRA }}$ and iterative receiving structure consisting of a serial concatenation of detector $(\mathrm{DET})$, convolutional decoder $\mathcal{D}_{\text {Acc }}$ and repetition decoder $\mathcal{D}_{\text {Rep }}$.

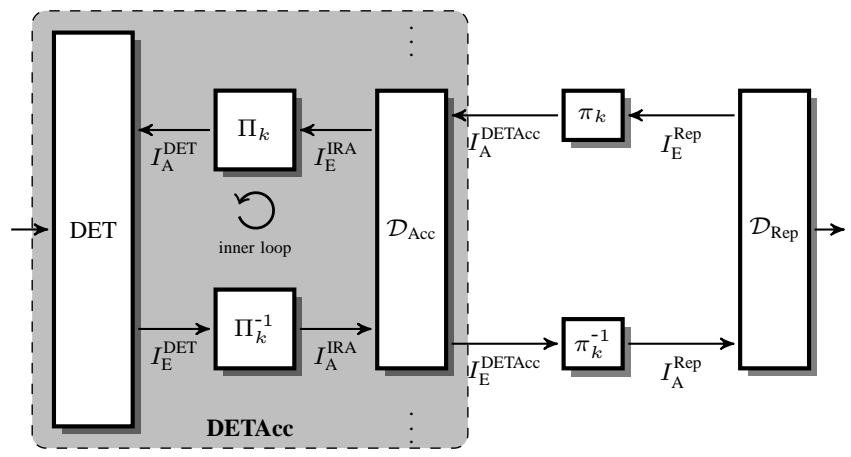

Fig. 2. Conventional approach: iterative receiver combining detector and convolutional decoder to inner entity; irregular repetition code is adapted to inner entity.

a set of inner setpoints finally allows to adapt the repetition code also to the convolutional decoder.

The remainder of this paper is structured as follows. In Sec. II the system model in described. In Sec. III the conventional as well as the proposed setpoint based IRA code design process are explained in detail. Also a concrete design example illustrating the fundamental differences between both approaches is given. Finally, Sec. IV concludes this paper.

\section{System Model}

A system as depicted in Fig. 1 is considered. $K$ information sequences $\mathbf{b}_{k}, 1 \leq k \leq K$ are encoded independently by the same IRA code $\mathcal{C}_{\text {IRA }}$. The IRA code consists of a serial concatenation of an irregular repetition code $\mathcal{C}_{\text {Rep }}$, a sequence specific interleaver $\pi_{k}$ and an accumulator $\mathcal{C}_{\text {Acc. }}$. The encoded sequences $\mathbf{c}_{k}$ are interleaved by sequence specific interleavers $\Pi_{k}$ resulting in the interleaved sequences $\mathbf{c}_{k}^{\prime}$ which are mapped to complex-valued symbols from an alphabet $\mathcal{A}$ and transmitted over a channel $\mathbf{H}$. This could, e.g, be a Multiple Access Channel (MAC) or a Multiple-Input MultipleOutput (MIMO) channel. No further assumptions regarding the mapping or the channel are made here. However, it is assumed that the detector (DET) at the destination is able to deliver Log-Likelihood-Ratios (LLRs) $\mathbf{L}_{c_{k}^{\prime}}^{\mathrm{DET}}$ of the transmitted interleaved code sequences $\mathbf{c}_{k}^{\prime}$.

The LLRs $\mathbf{L}_{c_{k}^{\prime}}^{\mathrm{DET}}$ are deinterleaved by $\Pi_{k}^{-1}$ and fed to the channel decoder which consists of a serial concatenation of a convolutional decoder $\mathcal{D}_{\text {Acc }}$ carried out as BCJR decoder
[9] and a repetition decoder $\mathcal{D}_{\text {Rep }}$. Now, an iterative detection process is invoked. Since three entities, namely the detector, the convolutional decoder and the repetition decoder exchange information, the question arises, how the information exchange should be scheduled. The conventional approach [4] is to combine the detector and the convolutional decoder to an inner loop whose joint transfer characteristic can be evaluated numerically by, e.g., EXIT-analysis [5]. The inner loop then regularily exchanges information with the repetition decoder in an outer loop. This approach is briefly recapped in Sec. III-A. For a more detailed description refer to, e.g., [4].

A more intuitive approach is to to keep the IRA decoding structure consisting of convolutional decoder and repetition decoder intact as an inner entity and to perform an outer detection loop between IRA decoder and detector. Clearly, since the detector as well as the convolutional decoder have to be taken into account when designing the irregular repetition code, this leads to a completely different design criterion for the irregular repetition code. In Sec. III-B such a design approach will be presented.

\section{IRA CODE DESIGN}

The principal task when designing IRA codes is to match the analytically describable irregular repetition code to the transfer characteristic of the convolutional decoder at the desired working point. Since the transfer characteristic of the convolutional decoder cannot be described analitically, it has to be evaluated numerically, e.g. by EXIT-analysis. In the context of iterative detection and decoding systems another entity plays a role in the overall code design, namely the detector whose task usually is to perform soft Interference Cancellation (sIC) and deliver LLRs of the codebits which are then processed by the channel decoder.

\section{A. Conventional approach}

The conventional approach to include the detector in the code design process is to combine detector (DET) and convolutional decoder (Acc) to an inner entity (DETAcc) and to adapt the irregular repetition code to this entity [4] as depicted in Fig. 2. In Fig. 3 the transfer characteristic

$$
I_{\mathrm{E}}^{\text {DETAcc }}=\mathrm{T}^{\text {DETAcc }}\left(I_{\mathrm{A}}^{\text {DETAcc }}, \sigma_{\mathrm{n}}^{2}, N_{\mathrm{it}, \text { in }}\right)
$$




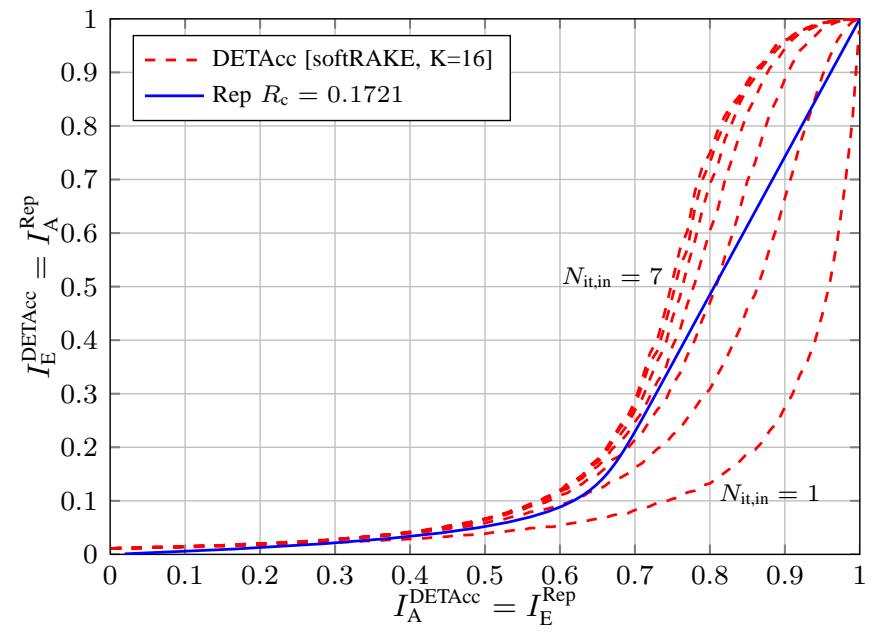

Fig. 3. Transfer characteristic of inner entity DETAcc consisting of detector and convolutional decoder for different numbers of inner iterations and optimized irregular repetition code. As example for the detector a soft-RAKE detector [10] for $K=16$ layers over an AWGN channel at $1 / \sigma_{\mathrm{n}}^{2}=5 \mathrm{~dB}$ is chosen.

of the inner loop is given for different numbers of inner iterations $N_{\mathrm{it}, \text { in }} . I_{\mathrm{A}}^{\mathrm{DETAcc}}$ hereby denotes the a-priori information which is fed back from the repetition decoder $\mathcal{D}_{\text {Rep }}$ and $\sigma_{\mathrm{n}}^{2}$ is the noise variance on the channel, i.e. the working point of the system. As can be seen from the figure, the transfer characteristic of the inner entity does not change significantly anymore after $N_{\text {it,in }}=5$ inner iterations. Hence, $N_{\text {it,in }}=5$ inner iterations are sufficient and the irregular repetition code should be adapted to this transfer characteristic.

The overall transfer characteristic $\mathrm{T}^{\mathrm{Rep}}$ of the irregular repetition code is given by

$$
I_{\mathrm{E}}^{\mathrm{Rep}}=\mathrm{T}^{\mathrm{Rep}}\left(I_{\mathrm{A}}^{\mathrm{Rep}}\right)=\sum_{\rho=\rho_{\min }}^{\rho_{\max }} w_{\rho} I_{\mathrm{E}, \rho}^{\mathrm{Rep}},
$$

where

$$
I_{\mathrm{E}, \rho}^{\mathrm{Rep}}=\mathrm{T}_{\rho}^{\mathrm{Rep}}\left(I_{\mathrm{A}, \rho}^{\mathrm{Rep}}, \rho\right)
$$

is the regular repetition code of code rate $R_{\mathrm{c}}=\frac{1}{\rho}$ and $0 \leq w_{\rho} \leq 1$ is its weight in the code mixture. Here, $\rho_{\text {min }}$ and $\rho_{\max }$ are design parameters limiting the minimal and maximal repetition factors in order to, e.g., control the errorfloor behaviour of the code [8].

The transfer characteristic of the repetition code can be described analytically as

$$
I_{\mathrm{E}, \rho}^{\mathrm{Rep}}=\mathrm{T}_{\rho}^{\mathrm{Rep}}\left(I_{\mathrm{A}, \rho}^{\mathrm{Rep}}, \rho\right)=\mathcal{J}\left((\rho-1) \mathcal{J}^{-1}\left(I_{\mathrm{A}, \rho}^{\mathrm{Rep}}\right)\right)
$$

with

$$
\begin{aligned}
\mathcal{J}(\nu) & =\left(1-2^{-1.0605 \nu^{0.8935}}\right)^{1.1064} \\
\mathcal{J}^{-1}(\nu) & =\left(-1 / 1.0605 \log _{2}\left(1-\nu^{1 / 1.1064}\right)\right)^{1 / 0.8935}
\end{aligned}
$$

denoting the J-function and its inverse.

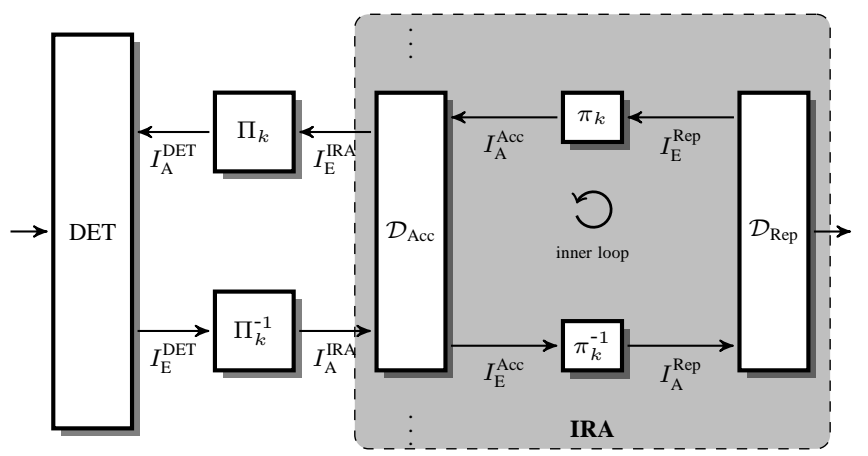

Fig. 4. Novel approach: iterative receiver combining convolutional decoder and repetition decoder to inner entity; irregular repetition code is adapted jointly to detector as well as convolutional decoder.

The goal is now to match the transfer characteristic $I_{\mathrm{E}}^{\text {Rep }}$ of the irregular repetition code to match the characteristic $I_{\mathrm{A}}^{\mathrm{DETAcc}}$ of the inner entity by adapting the weights $w_{\rho}$, i.e. to solve

$$
\begin{aligned}
& {\left[w_{\rho_{\min }}, \ldots, w_{\rho_{\max }}\right] }=\arg \min \left\{I_{\mathrm{E}}^{\text {Rep }}-I_{\mathrm{A}}^{\text {DETAcc }}+\Delta\right\} \\
& \text { s.t. } \sum_{\rho=\rho_{\min }}^{\rho_{\max }} w_{\rho}=1 \\
& I_{\mathrm{E}}^{\text {Rep }}>I_{\mathrm{A}}^{\text {DETAcc }},
\end{aligned}
$$

where $\Delta$ is the minimal allowed gap between both transfer curves and mainly determines the number of required iterations to achieve convergence.

The characteristic of the resulting irregular repetition code is depicted as well in Fig. 3. The code matches the characteristic of the inner entity very well up to approx. $I_{\mathrm{E}}^{\text {DETAcc }}=0.4$. Above this point, the s-curve behaviour of the inner entity which is mainly caused by the accumulator does not allow a perfect adaption of the repetition code. The coderate of the resulting overall code in this example is $R_{\mathrm{c}}=0.1721$.

\section{B. Setpoint-based approach}

A different approach aims at keeping the original decoding structure consisting of convolutional decoder and repetition decoder intact, i.e. performing the inner iterations between convolutional decoder and repetition decoder as depicted in Fig. 4. The outer iterations are then performed between the inner entity, i.e. the IRA channel decoder, and the detector. Since the irregular repetition code has to be adapted to both, the detector as well as the convolutional decoder, the inner, as well as the outer IRA code characteristic have to be considered in the design process.

1) Outer code behaviour: As a starting point, the desired outer IRA transfer characteristic

$$
\bar{I}_{\mathrm{E}}^{\mathrm{IRA}}=\mathrm{T}^{\mathrm{IRA}, \text { out }}\left(I_{\mathrm{A}}^{\mathrm{IRA}}\right)
$$

is formulated. It should be adapted to the transfer characteristic $I_{\mathrm{E}}^{\mathrm{DET}}=\mathrm{T}^{\mathrm{DET}}\left(I_{\mathrm{A}}^{\mathrm{DET}}, \sigma_{\mathrm{n}}^{2}\right)$ of the detector at the given working point $\sigma_{\mathrm{n}}^{2}$. Since the IRA code should match the detector as well as possible, the desired code characteristic is just set as the detector's characteristic with a fixed gap of $\Delta$ as 


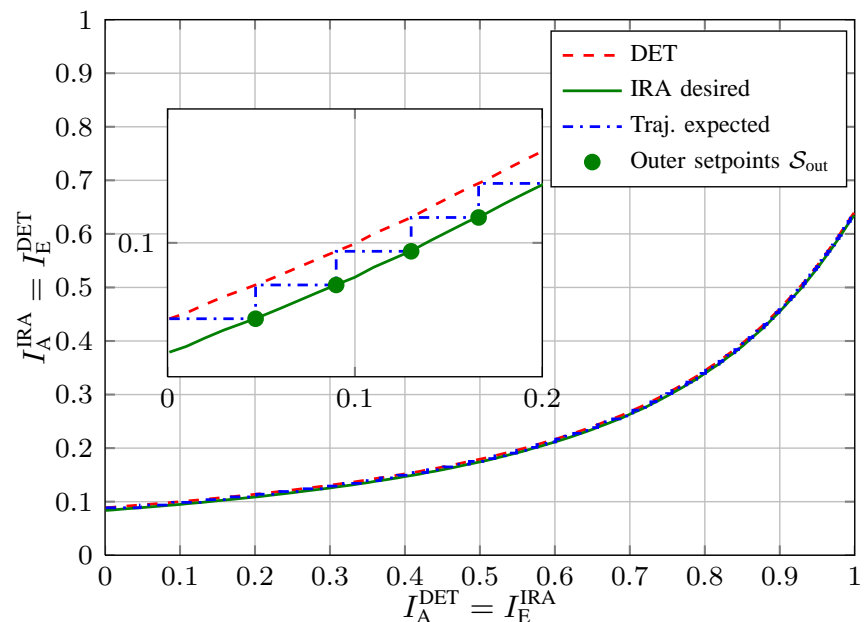

Fig. 5. Transfer characteristic of the detector at the working point (DET), the desired IRA code characteristic (IRA desired), the corresponding expected Trajectory (Traj. expected) and outer setpoints $s_{\text {out }, i} \in \mathcal{S}_{\text {out }}$.

$$
\bar{I}_{\mathrm{E}}^{\mathrm{IRA}}:=I_{\mathrm{A}}^{\mathrm{DET}}+\Delta
$$

as depicted in Fig. 5. From the desired code characteristic, an expected trajectory through the EXIT-chart can be determined as also shown in the figure. Sampling the desired code characteristic

$$
\bar{I}_{\mathrm{E}}^{\mathrm{IRA}}=\mathrm{T}^{\mathrm{IRA}, \text { out }}\left(I_{\mathrm{A}}^{\mathrm{IRA}}\right)
$$

at discrete points leads to a set $\mathcal{S}_{\text {out }}$ of outer setpoints

$$
s_{\text {out }, i}=\left(\bar{I}_{\mathrm{A}, i}^{\mathrm{IRA}} \bar{I}_{\mathrm{E}, i}^{\mathrm{RRA}}\right) \in \mathcal{S}_{\text {out }}
$$

which the IRA code should fulfill. This means for a given a-priori information $\bar{I}_{\mathrm{A}, \mathrm{i}}^{\mathrm{RA}}$ which is fed from the detector to the IRA decoder, the decoder has to generate extrinsic information $\bar{I}_{\mathrm{E}, i}^{\mathrm{IRA}}$ in order to achieve the desired characteristic. Achieving more extrinsic information than $\bar{I}_{\mathrm{E}, i}^{\mathrm{IRA}}$ means a rate loss [11] while achieving less might close the tunnel between detector and decoder and, thus, prohibit successfull detection. Clearly, the number of setpoints should be sufficiently large in order to approximate the desired characteristic. Hence, here one setpoint at every intersection between desired code characteristic and expected trajectory is calculated as depicted in Fig. 5.

2) Inner code behaviour: Having set the desired outer IRA code characteristic by outer setpoints, now the inner corresponding code characteristic has to be determined. That means, an irregular repetition code has to be found that for every outer setpoint $s_{\text {out }, i}$ the corresponding extrinsic information $I_{\mathrm{E}}^{\mathrm{IRA}}$ is generated. For this, the set of outer setpoints $\mathcal{S}_{\text {out }}$ is transformed into a set of inner setpoints $\mathcal{S}_{\text {in }}$.

First, the inner as well as the outer transfer characteristic of the convolutional decoder are evaluated at the input information $\bar{I}_{\mathrm{A}, \mathrm{i}}^{\mathrm{IRA}}$ determined by $s_{\mathrm{out}, i}$

$$
\begin{aligned}
& I_{\mathrm{E}}^{\mathrm{Acc}}=\mathrm{T}^{\mathrm{IRA}, \text { in }}\left(I_{\mathrm{A}}^{\mathrm{Acc}}, \bar{I}_{\mathrm{A}, i}^{\mathrm{IRA}}\right) \\
& I_{\mathrm{E}}^{\mathrm{IRA}}=\mathrm{T}^{\mathrm{IRA}, \text { out }}\left(I_{\mathrm{A}}^{\mathrm{Acc}}, \bar{I}_{\mathrm{A}, i}^{\mathrm{IRA}}\right) .
\end{aligned}
$$

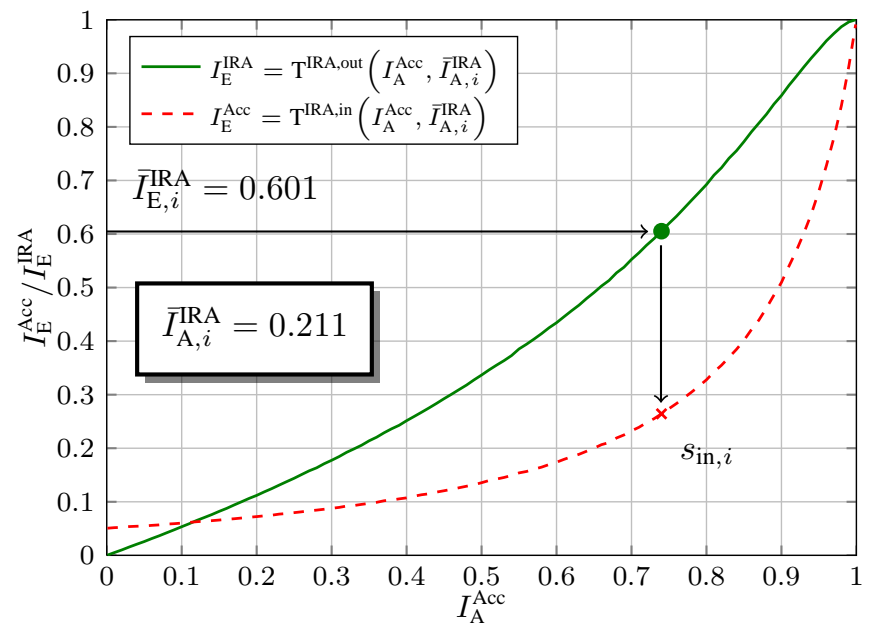

Fig. 6. Inner and outer transfer characteristics of the convolutional decoder $\mathcal{D}_{\text {Acc }}$ at working point $\bar{I}_{\mathrm{A}, i}^{\mathrm{IRA}}=0.211$. From the outer setpoint $s_{\text {out }, i}=(0.211,0.601)$ (green dot) the corresponding inner setpoint $s_{\text {in }, i}=$ $(0.211,0.264)$ (red cross) is found.

Here, $I_{\mathrm{E}}^{\text {Acc }}$ is the extrinsic information generated by the convolutional decoder with respect to its information bits at the given $\bar{I}_{\mathrm{A}, i}^{\mathrm{IRA}}$ and $I_{\mathrm{E}}^{\mathrm{IRA}}$ is the extrinsic information with respect to its codebits. From the outer setpoint $s_{\text {out }, i}$ the desired $\bar{I}_{E}^{\text {IRA }}$ is known which is indicated in Fig. 6 by a green dot. Mapping this point to the inner code characterisic

$$
\left(\bar{I}_{\mathrm{A}, i}^{\mathrm{IRA}}, \overline{I_{\mathrm{E}, i}^{\mathrm{RA}}}\right) \rightarrow \bar{I}_{\mathrm{E}, i}^{\mathrm{Acc}}
$$

directly gives the corresponding inner setpoint

$$
s_{\mathrm{in}, i}=\left(\bar{I}_{\mathrm{A}, i}^{\mathrm{Acc}}, \bar{I}_{\mathrm{E}, i}^{\mathrm{Acc}}\right) \in \mathcal{S}_{\text {in }} .
$$

This means, that the IRA code has to generate extrinsic information with respect to the information bits of $\bar{I}_{\mathrm{E}, i}^{\mathrm{Acc}}$ in order to produce extrinsic information of $\bar{I}_{\mathrm{E}, i}^{\mathrm{IRA}}$ with respect to the codebits, i.e., as feedback to the detector. Performing the above described procedure for every outer setpoint $s_{\text {out }, i} \in \mathcal{S}_{\text {out }}$ leads to the complete set of inner setpoints $s_{\text {in }, i} \in \mathcal{S}_{\text {in. }}$. In Fig. 7 all inner setpoints are plotted. They now describe the inner characteristic the irregular repetition code as to be adapted to in order to achieve the outer desired characteristic (7). Thus, the problem to be solved is

$$
\begin{aligned}
& {\left[w_{\rho_{\min }}, \ldots, w_{\rho_{\max }}\right] }=\arg \min \left\{I_{\mathrm{E}}^{\mathrm{Rep}}-\bar{I}_{\mathrm{A}}^{\mathrm{Acc}}\right\} \\
& \text { s.t. } \sum_{\rho=\rho_{\min }}^{\rho_{\max }} w_{\rho}=1 \\
& I_{\mathrm{E}}^{\mathrm{Rep}} \geq \bar{I}_{\mathrm{A}}^{\mathrm{Acc}},
\end{aligned}
$$

Note that in contrast to the conventional approach no gap $\Delta$ between convolutional decoder and repetition decoder characteristic is required as decoding should intentionally get stuck at the setpoints. In Fig. 7 also the resulting repetition code and as comparison the repetition code from the conventional design approach is given. Interestingly, both repetition codes have a very similar transfer characteristic and differ only slightly in code rate. 


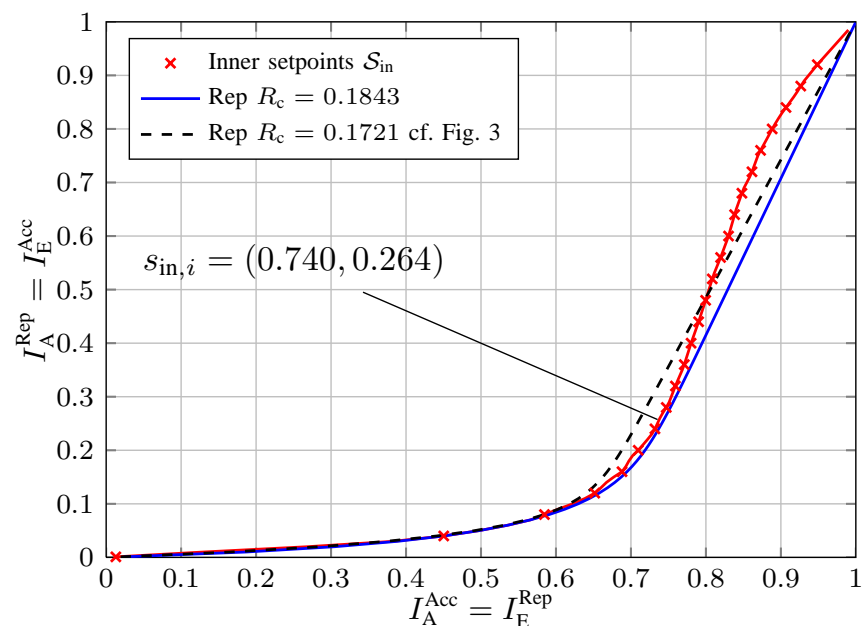

Fig. 7. The inner setpoints (red crosses) determine the overall characteristic the repetition code has to be adapted to. In contrast to Fig. 3 no gap between both curves is required.

In Fig. 8 again the transfer characteristic of the detector as well as the desired transfer characteristic of the IRA code are depicted. Furthermore, samples from the actual detection process obtained by Monte-Carlo simulations are shown. Each of these samples was obtained after the inner detection loop. As can be seen, the actual code characteristic follows the desired characteristic very closely. However, towards the end of the overall detection process, i.e. $I_{\mathrm{A}}^{\mathrm{IRA}} \gtrsim 0.25$, the code performance is better than desired. This is again due to the s-curve of the characteristic given by the inner setpoints as shown in Fig. 6, similar to the conventional design approach.

\section{CONCLUSION}

In this paper, a novel setpoint-based design approach for Irregular Repeat Accumulate (IRA) Codes in iterative detection and decoding systems was presented. The goal behind this approach is to keep the IRA decoding structure consisting of convolutional decoder and repetition decoder intact, i.e. to consider it as an inner loop of the overall detection structure. This requires the joint adaptation of the repetition code to the detector as well as to the convolutional decoder which was achieved by formulating a desired IRA code characteristic and defining a set of corresponding outer setpoints. Mapping these setpoints to a set of inner setpoints finally allowed the optimization of the desired repetition code. It was shown, that this approach, although starting from a completely different viewpoint than the conventional strategy, leads to an irregular repetition code with a very similar transfer characteristic and code rate as the conventional approach. However, the presented approach has a slightly higher computational complexity than the convential approach, since here usually more inner iterations are performed between convolutional decoder and repetition decoder as for the conventional approach between convolutional decoder and detector.

\section{REFERENCES}

[1] C. Berrou, A. Glavieux, and P. Thitimajshima, "Near Shannon Limit Error-Correcting Coding and Decoding: Turbo-Codes," in IEEE Inter-

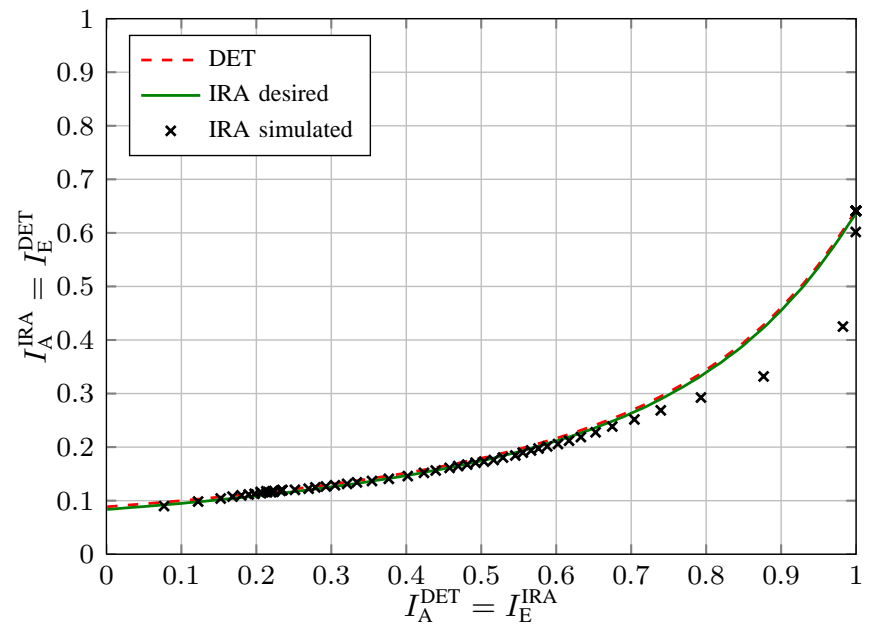

Fig. 8. Numerical results from Monte-Carlo simulations of the designed IRA code. IDMA multi-user system with $K=16$ users and soft-RAKE detection over AWGN channels at $1 / \sigma_{\mathrm{n}}^{2}=5 \mathrm{~dB}$. $N_{\mathrm{it}, \text { in }}=20$ inner Iterations and $N_{\text {it,out }}=50$ outer iterations.

national Communications Conference (ICC '93), Geneva, Switzerland, May 1993.

[2] D. Divsalar, H. Jin, and R. J. McEliece, "Coding Theorems for "TurboLike" Codes," in Proc. 36th Allerton Conference on Communication, Control, Computing, Allerton, IL, USA, Sep. 1998, pp. 201-210.

[3] H. Jin, A. Khandekar, and R. McEliece, "Irregular Repeat Accumulate Codes," in Second International Symposium on Turbo Codes, Brest, France, Sep. 2000.

[4] S. ten Brink and G. Kramer, "Design of Repeat-Accumulate Codes for Iterative Detection and Decoding," IEEE Transactions on Signal Processing, vol. 51, no. 11, pp. 2764-2772, Nov. 2003.

[5] S. ten Brink, "Convergence of Iterative Decoding," IEEE Electronic Letters, vol. 35, no. 13, pp. 1117-1119, May 1999.

[6] R. Zhang, L. Xu, S. Chen, and L. Hanzo, "Repeat Accumulate Code Division Multiple Access and its Hybrid Detection," in IEEE International Conference on Communication (ICC'08), Beijing, China, May 2008, pp. 4790-4794.

[7] F. Lenkeit, C. Bockelmann, D. Wübben, and A. Dekorsy, "IRA Code Design for IDMA-based Multi-Pair Bidirectional Relaying Systems," in Accepted for publication at 9th International Workshop on Broadband Wireless Access co-located with IEEE Globecom 2013, Atlanta, GA, USA, Dec. 2013.

[8] S. Johnson, Iterative Error Correction: Turbo, Low-Density ParityCheck and Repeat-Accumulate Codes. Cambridge University Press, 2009.

[9] L. Bahl, J. Cocke, F. Jelinek, and J. Raviv, "Optimal Decoding of Linear Codes for Minimizing Symbol Error Rate," IEEE Transactions on Information Theory, vol. 20, no. 2, pp. 284-287, Mar. 1974.

[10] L. Ping, L. Liu, K. Wu, and W. Leung, "Interleave-Division MultipleAccess," IEEE Transactions on Wireless Communications, vol. 5, no. 4, pp. 938-947, Apr. 2006.

[11] A. Ashikhmin, G. Kramer, and S. ten Brink, "Extrinsic Information Transfer Functions: Model and Erasure Channel Properties," IEEE Transactions on Information Theory, vol. 50, no. 11, pp. 2657-2673, Nov. 2004. 